PROCEEDINGS OF THE

AMERICAN MATHEMATICAL SOCIETY

Volume 129, Number 8, Pages 2365-2372

S 0002-9939(01)05971-8

Article electronically published on January 17, 2001

\title{
INVARIANT SUBSPACES FOR BOUNDED OPERATORS WITH LARGE LOCALIZABLE SPECTRUM
}

\author{
BEBE PRUNARU
}

(Communicated by Joseph A. Ball)

\begin{abstract}
Suppose $H$ is a complex Hilbert space and $T \in L(H)$ is a bounded operator. For each closed set $F \subset \mathbf{C}$ let $H_{T}(F)$ denote the corresponding spectral manifold. Let $\sigma_{l o c}(T)$ denote the set of all points $\lambda \in \sigma(T)$ with the property that $H_{T}(\bar{V}) \neq 0$ for any open neighborhood $V$ of $\lambda$. In this paper we show that if $\sigma_{l o c}(T)$ is dominating in some bounded open set, then $T$ has a nontrivial invariant subspace. As a corollary, every Hilbert space operator which is a quasiaffine transform of a subdecomposable operator with large spectrum has a nontrivial invariant subspace.
\end{abstract}

The main purpose of this paper is to give a certain spectral criterion for the existence of invariant subspaces for bounded operators on Hilbert spaces. Roughly speaking, if the spectrum of some operator $T$ can be localized in sufficiently many points, then a certain version of the dual algebras techniques initiated by S. Brown in Br1] can be successfully employed in this context, thus yielding nontrivial invariant subspaces.

The first application of the dual algebras techniques to operators having extensions with rich local spectral theory was made by C. Apostol in Ap. Subsequently, using a powerful result of M. Putinar [P], S. Brown [Br2] proved that all hyponormal operators with large spectrum have invariant subspaces. Eventually, invariant subspaces have been constructed for all Banach space operators with Bishop's property $(\beta)$ and large spectrum $[\mathrm{EPr}$. In all these cases, it was assumed that the operators under study have an extension or a lifting which are decomposable in the sense of C. Foiaş (see [CF]). We will replace this assumption with a certain localization property for the spectrum, which in particular is shared by all operators having a decomposable lifting.

We begin with several basic definitions and notations. Let $X$ be a complex Banach space, and let $L(X)$ denote the algebra of all bounded linear operators on $X$. For an open set $\omega \subset \mathbf{C}$, one denotes by $O(\omega, X)$ the Frechet space of all $X$ valued, analytic functions on $\omega$. Let us fix an operator $T \in L(X)$. For each closed set $F \subset \mathbf{C}$, we will denote by $X_{T}(F)$ the linear manifold of all vectors $x \in X$ such that $(z-T) u(z)=x, z \in \mathbf{C} \backslash F$, for some function $u \in O(\mathbf{C} \backslash F, X)$. For an open set $G \subset \mathbf{C}$, let $X_{T}(G)$ denote the union of all spaces $X_{T}(K)$, where $K$ runs through all compact subsets of $G$. In the case when $T$ has the single-valued extension property,

Received by the editors December 14, 1999.

2000 Mathematics Subject Classification. Primary 47A15, 47B20; Secondary 47A11, 47L45.

Key words and phrases. Invariant subspaces, local spectral theory.

The author was partially supported by Grant 5232/1999 from ANSTI.

(C)2001 American Mathematical Society 
the local spectrum of a vector $x \in X$, denoted by $\sigma_{T}(x)$, is defined as the smallest compact set $K$ with $x \in X_{T}(K)$. In this case, the local resolvent of $x$ is the unique function $u \in O\left(\mathbf{C} \backslash \sigma_{T}(x), X\right)$ satisfying $(z-T) u(z)=x, z \in \mathbf{C} \backslash \sigma_{T}(x)$. Also, recall that an operator $T \in L(X)$ is said to have Bishop's property $(\beta)$ if the map

$$
f \mapsto(z-T) f
$$

is one-to-one and has closed range on $O(\omega, X)$ for any open set $\omega \subset \mathbf{C}$. Moreover, $T$ is said to have property $(\delta)$ if $X=X_{T}(\bar{V})+X_{T}(\bar{W})$, whenever $\{V, W\}$ is an open cover of $\sigma(T)$.

As shown in [AE], an operator $T \in L(X)$ has property $(\beta)$ iff it is similar to the restriction of a decomposable operator to an invariant subspace. Similarly, $T$ has property $(\delta)$ iff it is the quotient of a decomposable operator. Moreover, properties $(\beta)$ and $(\delta)$ are dual to each other, in the sense that an operator $T \in L(X)$ has property $(\beta)$ iff its adjoint has property $(\delta)$, and conversely, $T$ has property $(\delta)$ iff its adjoint has property $(\beta)$.

For basic facts from local spectral theory, we refer to $[\mathrm{CF}$ ] (see also [AE] and EP] for recent advances in this field). For basic definitions and terminology in the theory of dual algebras see $[\mathrm{BFP}$.

Definition 1. Let $X$ be a complex Banach space and let $T \in L(X)$ be a bounded operator. Then the localizable spectrum $\sigma_{l o c}(T)$ of $T$ will be defined as the set of all $\lambda \in \mathbf{C}$ such that $X_{T}(\bar{V}) \neq 0$ for every open neighborhood $V$ of $\lambda$.

It is obvious that $\sigma_{l o c}(T)$ is a compact subset of the spectrum $\sigma(T)$ of $T$. In the case when $T$ has property $(\delta)$ one can easily see that $\sigma_{l o c}(T)=\sigma(T)$. In particular, $\sigma(T)=\sigma_{l o c}(T)$ whenever the adjoint $T^{*}$ is hyponormal.

Suppose now that $T$ and $S$ are bounded operators, and $T X=X S$ for some bounded one-to-one operator. Then one can show immediately that $\sigma_{l o c}(S) \subset$ $\sigma_{l o c}(T)$. In particular, $\sigma_{l o c}(S)=\sigma_{l o c}(T)$ whenever $T$ and $S$ are quasisimilar.

The main result of this paper is the following:

Theorem 1. Suppose $T \in L(H)$ is a bounded operator on a Hilbert space $H$ such that $\sigma_{l o c}(T) \cap \Omega$ is dominating in some bounded open set $\Omega$. Then $T$ has a nontrivial invariant subspace.

The following corollary is an immediate consequence of Theorem 1 and the remarks preceding it.

Corollary 1. Let $S$ be a bounded operator on some Banach space $X$ satisfying the following two conditions:

(i) $S$ has Bishop's property $(\beta)$, and

(ii) $\sigma(S) \cap \Omega$ is dominating in some bounded open set $\Omega \subset \mathbf{C}$.

Suppose moreover that $T \in L(H)$ is a Hilbert space operator such that $S X=X T$ for some dense range bounded operator $X: H \rightarrow X$. Then $T$ has a nontrivial invariant subspace.

The previous corollary enables us to give an alternate proof of the following recent result obtained in $\mathrm{AMcC}$ :

Corollary 2. Let $H$ and $H_{1}$ be complex Hilbert spaces, let $S \in L(H)$ be a nonnormal subnormal operator, and let $T \in L\left(H_{1}\right)$ such that $S X=X T$ for some dense range bounded operator $X: H_{1} \rightarrow H$. Then $T$ has a nontrivial invariant subspace. 
Proof. Let $\operatorname{Rat}(K)$ denote the space of all rational functions with poles outside some planar compact set $K$. Since $S$ is subnormal, its spectrum $\sigma(S)$ is a spectral set for $S$, i.e.

$$
\|f(S)\| \leq \sup \{|f(z)| ; z \in \sigma(S)\}
$$

for every $f \in \operatorname{Rat}(\sigma(S))$. Now, if $\operatorname{Rat}(\sigma(S))$ is norm-dense in the space $C(\sigma(S))$ of all continuous functions on $\sigma(S)$, then a result of J. von Neumann (see [Co] ) implies that $S$ is normal. This contradicts our initial assumption that $S$ is not normal. Therefore $\operatorname{Rat}(\sigma(S))$ is not dense in $C(\sigma(S))$, and in this case one knows (see $[\mathrm{Br} 2]$ ) that $\sigma(S)$ is dominating in some bounded open set $\Omega$. By Corollary 1 , $T$ has a nontrivial invariant subspace.

The proof of Theorem 1 is based on several lemmas and, before going further, we need to introduce some notation. For a bounded open set $\Omega \subset \mathbf{C}$, one denotes, as usual, by $H^{\infty}(\Omega)$ the Banach algebra of all bounded analytic functions on $\Omega$, endowed with the supnorm. A subset $S \subset \Omega$ is said to be dominating in $\Omega$ if $\sup _{z \in S}|f(z)|=\|f\|_{\infty}$ for any function $f \in H^{\infty}(\Omega)$. We will denote by $Q_{\Omega}$ the canonical predual of $H^{\infty}(\Omega)$, and for any point $\lambda \in \Omega$, we denote by $\phi_{\lambda}$ the corresponding evaluation at $\lambda$ which obviously belongs to $Q_{\Omega}$. In the next definition, $T \in L(H)$ is a fixed operator with the single valued extension property, and $\Omega \subset \mathbf{C}$ is a bounded open set.

Definition 2. For each pair of vectors $x \in H_{T}(\Omega)$ and $y \in H$, we define a bounded linear functional $[x \otimes y]$ on $H^{\infty}(\Omega)$ as follows:

$$
\langle f,[x \otimes y]\rangle=\frac{1}{2 \pi i} \int_{\gamma} f(\zeta)\left(x_{T}(\zeta), y\right) d \zeta, \quad f \in H^{\infty}(\Omega),
$$

where $x_{T}(\zeta)$ is the local resolvent of $x$ and $\gamma$ is a path in $\Omega$ surrounding $\sigma_{T}(x)$.

It is not difficult to see that the definition of $[x \otimes y]$ does not depend upon the particular choice of the path $\gamma$, as long as it satisfies the above conditions. Moreover, $\langle f,[x \otimes y]\rangle=(f(T) x, y)$ for any function $f \in H^{\infty}(\Omega)$ which is also analytic in a neighborhood of $\sigma(T)$ (in particular for any analytic polynomial). It is also obvious that $[x \otimes y]$ is a weak* continuous functional on $H^{\infty}(\Omega)$; hence it can be identified with a unique element in the predual $Q_{\Omega}$ of $H^{\infty}(\Omega)$.

Definition 3. Given $T \in L(H)$ with the single valued extension property, and a bounded open set $\Omega \subset \mathbf{C}$, let $X^{\Omega}(T)$ denote the set of all elements $\psi$ in the predual $Q_{\Omega}$ of $H^{\infty}(\Omega)$ for which there exists a sequence of vectors $\left\{x_{n}\right\}_{n=1}^{\infty}$ in the unit ball of $H_{T}(\Omega)$ satisfying the following three conditions:

$(\alpha) \lim _{n \rightarrow \infty}\left\|\psi-\left[x_{n} \otimes x_{n}\right]\right\|=0$

( $\beta) \lim _{n \rightarrow \infty}\left\|\left[x_{n} \otimes y\right]\right\|=0$ for all $y \in H$;

$(\gamma) \lim _{n \rightarrow \infty}\left\|\left[w \otimes x_{n}\right]\right\|=0$ for all $w \in H_{T}(\Omega)$.

For each $\lambda \in \mathbf{C}$ and $\rho>0$, one denotes by $D_{\rho}(\lambda)$ the open disc of radius $\rho$ centered at $\lambda$, and by $\bar{D}_{\rho}(\lambda)$ its closure. The following lemma is basic in the proof of Theorem 1 .

Lemma 1. Suppose $T \in L(H)$ is a bounded operator with empty point spectrum and $\Omega \subset \mathbf{C}$ is a bounded open set. Then for each $\lambda \in \sigma_{l o c}(T) \cap \Omega$ (if any) there exists a sequence of points $\left\{\mu_{n}\right\}_{n=1}^{\infty}$ in $\Omega$ such that $\mu_{n} \rightarrow \lambda$ and $\phi_{\mu_{n}} \in X^{\Omega}(T)$ for all $n \geq 1$. 
Proof. Let us fix $\lambda \in \sigma_{\text {loc }}(T) \cap \Omega$, let $\rho>0$ such that $D_{\rho}(\lambda) \subset \Omega$, and let $0<\epsilon<\frac{1}{3} \rho$. It then follows from the definition of $\sigma_{l o c}(T)$ that there exists a nonzero vector $x \in H$ such that $\sigma_{T}(x) \subset \bar{D}_{\epsilon}(\lambda)$. Let us denote by $H_{\epsilon}^{\lambda}$ the set of all functions $u \in O\left(\mathbf{C} \backslash \bar{D}_{\epsilon}(\lambda), H\right)$ for which there exists a vector $x \in H$ such that $\sigma_{T}(x) \subset \bar{D}_{\epsilon}(\lambda)$ and $u(\zeta)=x_{T}(\zeta)$ for all $\zeta \in \mathbf{C} \backslash \bar{D}_{\epsilon}(\lambda)$. We observe that if $u \in H_{\epsilon}^{\lambda}$ and $\mu \in \mathbf{C} \backslash \bar{D}_{\epsilon}(\lambda)$, then $\sigma_{T}(u(\mu)) \subset \bar{D}_{\epsilon}(\lambda)$. On $H_{\epsilon}^{\lambda}$ we define a norm as follows:

$$
\|u\|=\sup _{|\zeta-\lambda|=2 \epsilon}\|u(\zeta)\|, \quad u \in H_{\epsilon}^{\lambda}
$$

We denote by $\tilde{H}_{\epsilon}^{\lambda}$ the completion of $H_{\epsilon}^{\lambda}$ in this norm.

Therefore, $\tilde{H}_{\epsilon}^{\lambda}$ can be identified with the space of all continuous functions on the boundary $\partial D_{2 \epsilon}(\lambda)$ of $\bar{D}_{2 \epsilon}(\lambda)$ which are uniform limits on $\partial D_{2 \epsilon}(\lambda)$ of functions from $H_{\epsilon}^{\lambda}$. We also define a bounded operator $\tilde{T}$ on $\tilde{H}_{\epsilon}^{\lambda}$ by

$$
(\tilde{T} u)(\zeta)=T u(\zeta), \quad \zeta \in \partial D_{2 \epsilon}(\lambda),
$$

and a bounded operator $\tilde{q}: \tilde{H}_{\epsilon}^{\lambda} \rightarrow H$ by

$$
\tilde{q} u=\frac{1}{2 \pi i} \int_{\gamma_{\epsilon}} u(\zeta) d \zeta, \quad u \in \tilde{H}_{\epsilon}^{\lambda}
$$

where

$$
\gamma_{\epsilon}(t)=\lambda+2 \epsilon e^{i t}, \quad t \in[0,2 \pi) .
$$

We observe that if $u \in H_{\epsilon}^{\lambda}$ and $x \in H$ is a vector such that $(\zeta-T) u(\zeta)=x$ for all $\zeta \in \mathbf{C} \backslash \bar{D}_{\epsilon}(\lambda)$, then $\tilde{q} u=x$. In particular, this shows that the operator $\tilde{q}$ satisfies the identity $T \tilde{q}=\tilde{q} \tilde{T}$.

Now, our main purpose is to find a point $\mu \in D_{2 \epsilon}(\lambda)$ and a certain sequence of approximate eigenvectors $\left\{u_{n}\right\}_{n=1}^{\infty}$ for $\tilde{T}-\mu$, and then to show that $\phi_{\mu}$ and the sequence $\left\{x_{n}\right\}_{n=1}^{\infty}$ defined as $x_{n}=\tilde{q} u_{n}$ satisfy the three conditions defining the set $X^{\Omega}(T)$.

Our first claim in this direction is that there exists a point $\mu \in D_{2 \epsilon}(\lambda)$ such that the operator $\tilde{T}-\mu$ is not bounded below on $\tilde{H}_{\epsilon}^{\lambda}$. In order to prove this claim, first we show that the operator $\tilde{T}-\mu$ has dense range, for every $\mu$ with $|\mu-\lambda|>\epsilon$. Indeed, given such $\mu$ and a function $u \in H_{\epsilon}^{\lambda}$, the function $v$ defined as

$$
v(\zeta)=\frac{1}{\zeta-\mu}(u(\zeta)-u(\mu)), \quad \zeta \in \mathbf{C} \backslash \bar{D}_{\epsilon}(\lambda),
$$

satisfies $(T-\zeta) v(\zeta)=u(\mu)$ for all $\zeta \in \mathbf{C} \backslash \bar{D}_{\epsilon}(\lambda)$ so it belongs to the space $H_{\epsilon}^{\lambda}$, and moreover, $(\tilde{T}-\mu) v=u$. This easily implies that $\tilde{T}-\mu$ has dense range for any $\mu$ with $|\mu-\lambda|>\epsilon$.

Suppose now that $\tilde{T}-\mu$ is bounded below for any $\mu$ in $D_{2 \epsilon}(\lambda)$. It then follows from what we have already proved that $\tilde{T}-\mu$ is invertible for all $\mu$ with $\epsilon<|\mu-\lambda|<2 \epsilon$. Now, if there exists $\mu$ in $D_{2 \epsilon}(\lambda)$ such that $\tilde{T}-\mu$ is not invertible, then the boundary of the spectrum $\sigma(\tilde{T})$ intersects $D_{2 \epsilon}(\lambda)$; therefore the approximate point spectrum of $\tilde{T}$ intersects $D_{2 \epsilon}(\lambda)$ as well, which contradicts our previous assumption. Therefore, we see that, under the assumption that $\tilde{T}-\mu$ is bounded below for all $\mu \in D_{2 \epsilon}(\lambda)$, the operators $\tilde{T}-\mu$ are invertible for all such $\mu$. In this case, for each function $u \in H_{\epsilon}^{\lambda}$, the analytic function $\tilde{u}$ defined as

$$
\tilde{u}(\zeta)=\tilde{q}\left((\zeta-\tilde{T})^{-1} u\right), \quad|\zeta-\lambda|<2 \epsilon,
$$


satisfies the identity

$$
(\zeta-T) \tilde{u}(\zeta)=\tilde{q} u, \quad \zeta \in D_{2 \epsilon}(\lambda)
$$

thus it represents an analytic extension of $u$ in a neighborhood of $\bar{D}_{\epsilon}(\lambda)$. This implies that the local spectrum of $x=\tilde{q} u$ is empty, hence $x=0$. However, this contradicts our initial assumption that $\lambda \in \sigma_{l o c}(T)$. Therefore, our claim that for some $\mu$ in $D_{2 \epsilon}(\lambda), \tilde{T}-\mu$ is not bounded below, is completely proved.

We now fix such a point $\mu$ and also fix a sequence of unit vectors $\left\{u_{n}\right\}_{n=1}^{\infty}$ in $H_{\epsilon}^{\lambda}$ such that $\left\|(\tilde{T}-\mu) u_{n}\right\| \rightarrow 0$. Let $x_{n}=\tilde{q} u_{n}$. Since $T \tilde{q}=\tilde{q} \tilde{T}$, we easily see that $\left\|(T-\mu) x_{n}\right\| \rightarrow 0$. Moreover $\inf _{n}\left\|x_{n}\right\|>0$. Suppose, on the contrary, that $\left\|x_{n}\right\| \rightarrow 0$. In this case, the identity $(\zeta-T) u_{n}(\zeta)=x_{n}$ for $\zeta \in \mathbf{C} \backslash \bar{D}_{\epsilon}(\lambda)$ shows that $\left\|(\mu-\zeta) u_{n}(\zeta)\right\| \rightarrow 0$ uniformly on $\partial D_{2 \epsilon}(\lambda)$. Since $\mu$ is a point in $D_{2 \epsilon}(\lambda)$, this implies that $\left\|u_{n}\right\| \rightarrow 0$ in $H_{\epsilon}^{\lambda}$ which contradicts the fact that $\left\{u_{n}\right\}_{n=1}^{\infty}$ are unit vectors in $H_{\epsilon}^{\lambda}$. We may and shall assume that $\left\|x_{n}\right\|=1$, for all $n \geq 1$. In addition, since the point spectrum of $T$ is empty, we may assume that $x_{n} \rightarrow 0$ weakly. Our next task is to prove that the sequence $\left\{x_{n}\right\}_{n=1}^{\infty}$ satisfies the three conditions $(\alpha)-(\gamma)$.

Proof of $(\alpha)$. Let $f \in H^{\infty}(\Omega)$ with $\|f\|_{\infty}=1$ and write $f$ as

$$
f(\zeta)-f(\mu)=g(\zeta)(\zeta-\mu), \quad \zeta \in \Omega,
$$

with $\|g\|_{\infty} \leq 2\|f\|_{\infty} / \operatorname{dist}(\mu, \partial \Omega)$. Let $\chi(\zeta)=\zeta$ denote the position function in $H^{\infty}(\Omega)$, and recall that $\gamma_{\epsilon}$ is the path whose image coincides with the boundary of $\bar{D}_{2 \epsilon}(\lambda)$. Since $\sigma_{T}\left(x_{n}\right) \subset \bar{D}_{\epsilon}(\lambda)$, and $\left\|x_{n}\right\|=1$, the definition of $\left[x_{n} \otimes x_{n}\right]$ shows that

$$
\begin{gathered}
\left\langle f, \phi_{\mu}-\left[x_{n} \otimes x_{n}\right]\right\rangle \\
=\left\langle g(\chi-\mu),\left[x_{n} \otimes x_{n}\right]\right\rangle \\
=\frac{1}{2 \pi i} \int_{\gamma_{\epsilon}} g(\zeta)(\zeta-\mu)\left(u_{n}(\zeta), x_{n}\right) d \zeta \\
=\frac{1}{2 \pi i} \int_{\gamma_{\epsilon}} g(\zeta)\left((T-\mu) u_{n}(\zeta), x_{n}\right) d \zeta \\
=\frac{1}{2 \pi i} \int_{\gamma_{\epsilon}} g(\zeta)\left(\left((\tilde{T}-\mu) u_{n}\right)(\zeta), x_{n}\right) d \zeta .
\end{gathered}
$$

Therefore

$$
\left\|\phi_{\mu}-\left[x_{n} \otimes x_{n}\right]\right\| \leq 4\left\|(\tilde{T}-\mu) u_{n}\right\| \rightarrow 0,
$$

and the proof of $(\alpha)$ is complete.

Proof of $(\beta)$. As in the proof of $(\alpha)$, we choose a function $f \in H^{\infty}(\Omega)$ of norm one, and write $f$ as

$$
f(\zeta)-f(\mu)=g(\zeta)(\zeta-\mu), \quad \zeta \in \Omega,
$$

with $\|g\|_{\infty} \leq 2\|f\|_{\infty} / \operatorname{dist}(\mu, \partial \Omega)$. Therefore

$$
\begin{gathered}
\left\langle f,\left[x_{n} \otimes y\right]\right\rangle \\
=\left\langle g(\chi-\mu),\left[x_{n} \otimes y\right]\right\rangle+f(\mu)\left(x_{n}, y\right) .
\end{gathered}
$$


The definition of $\left[x_{n} \otimes y\right]$ shows that

$$
\begin{aligned}
& \left\langle g(\chi-\mu),\left[x_{n} \otimes y\right]\right\rangle \\
= & \frac{1}{2 \pi i} \int_{\gamma_{\epsilon}} g(\zeta)(\zeta-\mu)\left(u_{n}(\zeta), y\right) d \zeta \\
= & \frac{1}{2 \pi i} \int_{\gamma_{\epsilon}} g(\zeta)\left((T-\mu) u_{n}(\zeta), y\right) d \zeta \\
= & \frac{1}{2 \pi i} \int_{\gamma_{\epsilon}} g(\zeta)\left(\left((\tilde{T}-\mu) u_{n}\right)(\zeta), y\right) d \zeta
\end{aligned}
$$

Therefore

$$
\left\|\left[x_{n} \otimes y\right]\right\| \leq\left(4\left\|(\tilde{T}-\mu) u_{n}\right\|\|y\|+\left|\left(x_{n}, y\right)\right|\right) \rightarrow 0,
$$

and this concludes the proof of $(\beta)$.

Proof of $(\gamma)$. Let $w \in H_{T}(\Omega)$, let $\Gamma$ be a contour in $\Omega$ surrounding $\sigma_{T}(w)$, and let $w_{T}$ denote the local resolvent of $w$. Then one can easily see that the set of all vectors $t \in H$ of the form

$$
t=\frac{1}{2 \pi i} \int_{\Gamma} f(\zeta) w_{T}(\zeta) d \zeta,
$$

where $f$ runs through the unit ball of $H^{\infty}(\Omega)$, is a norm compact subset in $H$. Since $x_{n} \rightarrow 0$ weakly, we infer that $\lim _{n \rightarrow \infty}\left\|\left[w \otimes x_{n}\right]\right\|=0$. The conclusion is that the sequence $\left\{x_{n}\right\}_{n=1}^{\infty}$ satisfies all conditions $(\alpha)-(\gamma)$. The proof of this lemma is completed.

From now on, $T \in L(H)$ will be a bounded operator such that $\sigma_{l o c}(T) \cap \Omega$ is dominating in some bounded open set $\Omega$. We shall assume that the point spectrum of $T$ is empty; therefore $T$ has the single-valued extension property. The proofs of the following two lemmas are very similar to those appearing in $[\mathrm{Br} 1]$ or $[\mathrm{BCP}$, and they will be only sketched below.

Lemma 2. Let $\left\{\alpha_{1}, \ldots, \alpha_{r}\right\} \subset \mathbf{C},\left\{\lambda_{1}, \ldots, \lambda_{r}\right\} \subset \sigma_{l o c}(T) \cap \Omega, x_{0} \in H_{T}(\Omega), y_{0} \in$ $H, \epsilon>0$, and $\delta>0$ such that $\sum_{j=1}^{r}\left|\alpha_{j}\right|<\delta$. Then there exist vectors $\tilde{x} \in H_{T}(\Omega)$ and $\tilde{y} \in H$ such that:

(i) $\left\|\sum_{j=1}^{r} \alpha_{j} \phi_{\lambda_{j}}-[\tilde{x} \otimes \tilde{y}]\right\|<\epsilon$;

(ii) $\|\tilde{x}\|<\delta^{1 / 2}$;

(iii) $\|\tilde{y}\|<\delta^{1 / 2}$;

(iv) $\left\|\left[\tilde{x} \otimes y_{0}\right]\right\|<\epsilon$;

(v) $\left\|\left[x_{0} \otimes \tilde{y}\right]\right\|<\epsilon$.

Proof. Let $\eta>0$ be a sufficiently small number, to be specified later. Using Lemma 1 and the fact that the map

$$
\Omega \ni \mu \mapsto \phi_{\mu}
$$

is continuous, we can find inductively unit vectors $\left\{x_{1}, \ldots, x_{r}\right\} \subset H_{T}(\Omega)$ satisfying the following conditions:

(1) $\left\|\phi_{\lambda_{j}}-\left[x_{j} \otimes x_{j}\right]\right\|<\eta, \quad 1 \leq j \leq r$;

(2) $\left\|\left[x_{0} \otimes x_{j}\right]\right\|+\left\|\left[x_{j} \otimes y_{0}\right]\right\|<\eta, \quad 1 \leq j \leq r$;

(3) $\max _{1 \leq i, j \leq r, i \neq j}\left\{\left\|\left[x_{i} \otimes x_{j}\right]\right\|\right\}<\eta$. 
Let $\beta_{j} \in \mathbf{C}$ such that $\beta_{j}^{2}=\alpha_{j}$ for $1 \leq j \leq r$, and define $\tilde{x}=\sum_{j=1}^{r} \beta_{j} x_{j}$ and $\tilde{y}=\sum_{j=1}^{r} \bar{\beta}_{j} x_{j}$. It is now obvious that, for sufficiently small $\eta$, these two vectors satisfy conditions (i) $-(\mathrm{v})$. The proof of this lemma is complete.

Lemma 3. Let $\epsilon>0$, let $\psi$ be an element in the predual $Q_{\Omega}$, and let $x_{0} \in H_{T}(\Omega)$ and $y_{0} \in H$ be vectors such that $\|\psi-[x \otimes y]\|<\delta$, for some $\delta>0$. Then there exist vectors $x_{1} \in H_{T}(\Omega)$ and $y_{1} \in H$ such that:

(i) $\left\|\psi-\left[x_{1} \otimes y_{1}\right]\right\|<\epsilon$;

(ii) $\left\|x_{1}-x_{0}\right\|<\delta^{1 / 2}$;

(iii) $\left\|y_{1}-y_{0}\right\|<\delta^{1 / 2}$.

Proof. Let $\eta>0$ be a sufficiently small number. Since the set $\sigma_{l o c}(T) \cap \Omega$ is dominating in $\Omega$, the bipolar theorem shows that we can find a finite set of points $\left\{\lambda_{1}, \ldots, \lambda_{r}\right\} \subset \sigma_{\text {loc }}(T) \cap \Omega$ and complex scalars $\left\{\alpha_{1}, \ldots, \alpha_{r}\right\}$ such that $\sum_{j=1}^{r}\left|\alpha_{j}\right|<$ $\delta$ and

$$
\left\|\psi-\left[x_{0} \otimes y_{0}\right]-\sum_{j=1}^{r} \alpha_{j} \phi_{\lambda_{j}}\right\|<\eta .
$$

Now, according to Lemma 2 , we can find a pair of vectors $\tilde{x} \in H_{T}(\Omega)$ and $\tilde{y} \in H$ such that

(i) $\left\|\sum_{j=1}^{r} \alpha_{j} \phi_{\lambda_{j}}-[\tilde{x} \otimes \tilde{y}]\right\|<\eta$;

(ii) $\|\tilde{x}\|<\delta^{1 / 2}$;

(iii) $\|\tilde{y}\|<\delta^{1 / 2}$;

(iv) $\left\|\left[\tilde{x} \otimes y_{0}\right]\right\|<\eta$;

(v) $\left\|\left[x_{0} \otimes \tilde{y}\right]\right\|<\eta$.

It then follows that, for a sufficiently small $\eta>0$, the vectors $x_{1}=x_{0}+\tilde{x}$ and $y_{1}=y_{0}+\tilde{y}$ satisfy all three conditions (i)-(iii). This completes the proof of this lemma.

Proof of Theorem 1. Let us fix a point $\lambda \in \Omega$. It then follows from the previous lemma that there exist Cauchy sequences $\left\{x_{n}\right\}_{n=1}^{\infty} \subset H_{T}(\Omega)$ and $\left\{y_{n}\right\}_{n=1}^{\infty} \subset H$ such that $\left\|\phi_{\lambda}-\left[x_{n} \otimes y_{n}\right]\right\| \rightarrow 0$. Let $x=\lim x_{n}$ and $y=\lim y_{n}$. It then follows immediately that $p(\lambda)=(p(T) x, y)$ for any polynomial, which shows that $T$ has indeed a nontrivial invariant subspace. This completes the proof of Theorem 1.

\section{REFERENCES}

[AMcC] J. Agler and J. McCarthy, Operators that dominate normal operators, J. Operator Theory 40 (1998), 385-407. MR 99i:47038

[AE] E. Albrecht and J. Eschmeier, Analytic functional models and local spectral theory, Proc. London Math. Soc. 75 (1997), 323-345. MR 98f:47043

[Ap] C. Apostol, The spectral flavor of Scott Brown's techniques, J. Operator Theory 6 (1981), 3-12. MR 83a:47005a

[BFP] H. Bercovici, C. Foias and C. Pearcy, Dual Algebras with Applications to Invariant Subspaces and Dilation Theory, C.B.M.S. Regional Conf. Ser. in Math. no. 56, Amer. Math. Soc., Providence, R.I., 1985. MR 87g:47091

[Br1] S. Brown, Some invariant subspaces for subnormal operators, Integral Equations and Operator Theory 1 (1978), 310-333. MR 80c:47007

[Br2] Hyponormal operators with thick spectrum have invariant subspaces, Annals of Math. 125 (1987), 93-103. MR 88c:47010

[BCP] S. Brown, B. Chevreau, and C. Pearcy, Contractions with rich spectrum have invariant subspaces, J. Operator Theory 1 (1979), 123-136. MR 80m:47002 
[CF] I. Colojoara and C. Foias, The Theory of Generalized Spectral Operators, Gordon and Breach, New York, 1968. MR 52:15085

[Co] J.B. Conway, The Theory of Subnormal Operators, Amer. Math. Soc., Providence, RI, 1991. MR 92h:47026

[EPr] J. Eschmeier and B. Prunaru, Invariant subspaces for operators with Bishop's property ( $\beta$ ) and thick spectrum, J. Funct. Anal. 94 (1990), 196-222. MR 91m:47008

[EP] J. Eschmeier and M. Putinar, Spectral Decompositions and Analytic Sheaves, Oxford University Press, New York, 1996. MR 98h:47002

[P] M. Putinar, Hyponormal operators are subscalar, J. Operator Theory 12 (1984), 385-395. MR 85h: 47027

Institute of Mathematics, Romanian Academy, P.O. Box 1-764, 70700 Bucharest, RoMANIA

E-mail address: bprunaru@imar.ro 